\title{
Insurance with Frequent Trading
}

\author{
Jose S. Penalva Zuasti* \\ Universitat Pompen Fabra
}

First version: October, 1997

This version: March 01, 1999

${ }^{*}$ I would like to thank Bryan Ellickson and William R. Zame for long ours of helpful discussion and all their comments and suggestions. I also wish to thank audiences at Brown University, New York University, the William E. Simon Graduate School of Business, UC Los Angeles, UC Santa Cruz, Universitat Pompeu Fabra, Universidad Carlos III, and Universidad de Alicante for their helpful questions and comments. All errors are my own. 


\begin{abstract}
This paper looks at the dynamic management of risk in an economy with discrete time consumption and endowments and continuous trading. I study how agents in such an economy deal with all the risk in the economy and attain their Pareto optimal allocations by trading in a few natural securities: private insurance contracts and a common set of derivatives on the aggregate endowment. The parsimonious nature of the implied securities needed for Pareto optimality suggests that in such contexts complete markets is a very reasonable assumption.
\end{abstract}

Keywords: risk-sharing, insurance, hedging, point-processes, complete markets, general equilibrium

JEL Classification: D81, D99, G11 


\section{Introduction}

In January 1994 an earthquake of magnitude 6.7 shook Northridge, an urban neighborhood of Los Angeles, causing over 13 billion dollars in damage. The Northridge earthquake is one of a number of natural catastrophes to have hit the U.S. in the recent past; many more will surely occur in the near future. The risk presented by such phenomena has been analyzed from two different perspectives: insurance and finance. I propose to construct a model that allows me to combine both perspectives and better understand what it takes to manage risks dynamically and how plausible it is for markets to be complete.

From the insurance perspective, the main risk from an earthquake is that, given any fixed insurance premium, actual fluctuations in claims will at some point exceed the amount collected from the premia. From Malinvaud (1972, 1973), a significant part of the literature on insurance has dealt with how do insurance markets work despite this risk. Malinvaud showed that this risk is minimized in industries where there are a very large number of independent individual risks and where, as we know from the Law of Large Numbers (LLN), actual claims will differ very little from expected claims. Natural disasters pose a challenge because the individual risks being covered are intrinsically not independent. This problem is not exclusive to catastrophic insurance but is a generic property of economic situations in which there is a substantial component of risk in the aggregate. Cass, Chilchininsky, and Wu (1996) proposed a way to deal with this risk by having additional securities to deal with the aggregate component of risk as well as private insurance contracts. Ellickson and Penalva (1997) deal with this by allowing the possibility that accidents happen gradually as part of a process that unfolds over time.

The second approach is the finance perspective. From this perspective the risk posed by natural catastrophes is just another risk which appropriately securitized and traded can be dealt with through optimal portfolio diversification, as we know from Markowitz (1952) and the literature on the Capital Asset Pricing Model (CAPM, Breeden (1979), Duffie and Zame (1989)). From this perspective, earthquake risk is in no way different from the risk of biotechnological innovation or movie-making: it is just an unlikely event with a very high (or low) possible payoff. As such, it can easily be incorporated as a small proportion of a well diversified portfolio. The key

aspect from the finance point of view is that there be enough securities to deal with all the possible risks, i.e. markets are complete (Duffie and Huang (1985), Duffie and Zame (1989)). 
The approach I take in this paper builds on the insurance approach of Cass, Chilchininski, and Wu, and of Ellickson and Penalva that looks at the origin of the risk and its role at the level of the individual to extend the results of the optimal risk sharing literature (Wilson, Diamond, Mace, and Townsend) in the context of a continuous trading economy as in Duffie and Zame. The main result is a very parsimonious description of optimal trading behaviour, suggesting that markets will be complete.

\section{The Framework}

In this section I will describe the main elements that define the framework and introduce the economic primitives and necessary notation that I will use later to study how agents use markets to share risks.

\subsection{Hybrid Time}

I model time on two scales, a discrete one for consumption and a continuous one for security trading. This approach combines the benefits of existing continuous time and discrete time models in a single framework, while avoiding some of the problems with modeling time either as continuous or as discrete.

The approach I propose is a generalization of the framework first used to study continuous pricing and trading (see the survey by Merton [1990]). The framework they used is to have time defined over a finite interval. Consumption and endowments would take place at two discrete dates, the beginning (call it date 0 ) and the end of the period (date $T$ ), and security trading and pricing took place at any date within the period. These models describe uncertainty as a stochastic process on the interval between 0 and $T$. The great advantage of these models is that uncertainty is revealed gradually. This implies that agents can manage a great deal of uncertainty if they are allowed to trade often even if it is in a finite number of securities. Also, such models will have a Walrasian equilibrium and the equilibrium can be implemented as a security market equilibrium. This is because of the special properties of the VonNeumann-Morgenstern expected utility representation of preferences. On the practical side, these models have been very useful for pricing stocks and financial derivatives, such as options, which are traded continuously in the stock exchange.

The two date consumption model cannot be used to study dynamic economies. Among other things, such models are insufficient for the study of the term structure of interest rates. A solution is to allow agents to have endowments and consume at intermediate dates. New models were 
introduced in which agents had endowments and could consume at every intermediate date. These models have been extensively studied and used. But introducing consumption and endowments over continuous time poses serious problems for implementations, both in terms of the right preferences to use and the interpretation of endowments as flows of commodities that are totally ephemeral: commodities only exist during the instant in time at which they arrive in the economy.

These problems do not arise in models that describe time using a discrete scale. It is very easy to write down preferences for discrete time models which are relatively easy to work with and their welfare properties are wellunderstood. Also, endowments and commodities are very easy to interpret using existing data. These advantages explain why discrete time models are used to study many aspects of dynamic economies: growth, unemployment, investment, inflation, ... It is fair to say that discrete models are the main ingredient of down-to-earth applied work in economics. Unfortunately, it is very difficult to use these discrete time models to study pricing and trading in financial markets that are continuously open. Also, agents lack the

trading opportunities available with continuous time models and hence the assumption of complete markets is hard to justify.

In this paper I propose a particular way to combine discrete time with continuous time finance in a coherent way and in a way that takes advantage of the benefits of both continuous time finance models and discrete time economic ones. The key is to take advantage of the separation of the optimal consumption problem and the wealth allocation problem. With this, the economy can be defined on two time scales. The optimal consumption problem is defined in discrete time (via preferences and consumption commodities) and agents make their wealth allocation decisions in continuous time, via security markets that are open between consumption dates.

\subsection{Marked Point Processes}

The second main element I propose to use is Marked Point Processes (MPPs). MPPs have been substantially used in the finance literature to describe the dynamics of security prices and dividend process. I propose to use it as the way to model the fundamental, underlying uncertainty in the economy. Because security prices will reflect that uncertainty, their dynamics will be determined by the properties of the underlying MPP, but they will also be affected by economic considerations. This approach follows the tradition of the general equilibrium treatment of asset prices, and departs from the more pure mathematical approaches that take prices and price dynamics as given. 
As I have described in the introduction, the discrete nature of the processes I wish to study requires a different mathematical representation than the more usual Brownian Motion. That is why I use MPPs. MPPs are continuously revealing information but they differ from Brownian motion in that for the most part the information being revealed is of the form: "nothing new has happened'. MPPs have already been used in finance (Aase[1993], Cox and Ross[1976], Merton[1990]) but the approach I take is slightly different in that instead of assuming that prices or dividends follow a marked point process, I will assume that MPPs describe the primitive uncertainty in the economy. The reason I wish to focus on MPPs is two-fold: they are more practical and they are a better description of the kinds of shocks we observe in real economies.

MPPs are a generalization of Poisson processes. The classic example is the arrival of customers to a bank teller's window. A customer can arrive at the window at any point in time. Those random times are described by a Poisson process. MPPs behave in much the same way, but they generalize Poisson in two ways: first, the arrival rate does not have to be timeindependent, so that at any given time the probability of an arrival over the next time interval can depend on what has happened up until the time of the arrival; and second, MPPs allows coding additional information about the arrival, such as whether the customer wants to make a deposit, withdraw cash, open a new account, etc. The arrival times are called jump times and the additional information describing an arrival time is called the mark of the jump.

These properties of MPPs make them ideally suited for dealing with the kind of information arrival needed in practical economic applications. The main examples I will be working with in this paper are insurance markets. In such a context, the kind of information described by an MPP is the occurrence of an accident: a car accident, the diagnosis of a malignant tumor, an earthquake. In other contexts, the MPP can describe the discovery of a new technology, the destruction of a harvest by a particularly strong storm, changes in tax laws, the announcement of an interest rate increase, etc. The uncertainty surrounding these kinds of applications is particularly well suited to MPPs.

Also, MPPs are easier to work with than difussion processes like Brownian Motion. The stochastic calculus of MPPs uses standard LebesgueStieltjes integration, and the paths followed by an MPP have very intuitive descriptions: the path of a marked point process is entirely described by a list of the times at which a jump took place and the type of jump that took place. 
MPPs combined with hybrid time provide the essential elements with which to construct a theoretical framework that is both general and applicationfriendly.

\subsection{The Economic Primitives}

In this section I will present the formal description of the framework. It consists of a dynamic exchange economy with uncertainty, a single commodity ${ }^{1}$ at each date-event, and a finite number of agents.

Assumption $1 N$ is a MPP, where

$$
N(t)=\left(N_{1}(t), N_{2}(t), \ldots, N_{K}(t)\right),
$$

is a vector of counting functions, $N_{k}(t)$, describes the number of jumps of type $k$ that have taken place up to and including time $t \in \mathcal{T}:=[0, T]$ ( $T$ can be either finite or infinite). $N$ admits a bounded intensity, $\lambda$. Let $\Omega$ be the space of all possible paths of the MPP N. N describes a filtration on $\Omega$,

$$
\mathbf{F}:=\left(\mathcal{F}_{t}\right)_{t \in \mathcal{T}},
$$

where $\mathcal{F}_{t}:=\sigma(N(s), s \leq t)$, and $\mathcal{F}=\cup_{t \in \mathcal{T}} \mathcal{F}_{t}$. Let $P$ a probability measure on $(\Omega, \mathcal{F})$. The fundamental uncertainty in the economy is described by $(\Omega, \mathcal{F}, P, \mathbf{F})$.

This assumption describes the kind of uncertainty facing the economy. The main things to note are:

- Uncertainty is exogenous. This assumption is quite restrictive but is the necessary starting point. It precludes problems that involve moral hazard and adverse selection, but such issues are difficult for general equilibrium frameworks more generally.

- By assuming that $N$ admits an intensity, I am limiting the rate at which accidents can take place. The very notion of a rate is intimately linked to the intensity (see Bremaud[1981]).

- A bounded intensity means that accidents are spread out over $[0, T]$. This ensures that with probability one, the number of jumps in any finite time interval will be finite.

\footnotetext{
${ }^{1}$ The framework has been extended to deal with any finite number of commodities see Penalva[1997].

${ }^{2} \sigma(x(s), s \leq t)$ denotes the $\sigma$-algebra generated by the process $x(t)$ up to and including time $t$.
} 
The following definition will be useful later. Denote

$$
M_{k}(t):=N_{k}(t)-\int_{0}^{t} \lambda_{k}(s) d s
$$

as the canonical martingales ${ }^{3}$. A process $x(t)$ is a martingale with respect to measure $P$ and filtration $\left(\mathcal{F}_{t}\right)_{s>0}$, if it is measurable with respect of $\left(\mathcal{F}_{t}\right)_{s>t}$, $E_{P}[|x(t)|]<\infty$ for all $t$, and for all $s \leq t, E_{P}\left[x(t) \mid \mathcal{F}_{s}\right]=x(s) . M_{k}(t)$ is a $P$-martingale and the $M_{k}$ 's form a basis for the space of $P$-martingales ${ }^{4}$.

Assumption 2 The commodity space, $L$, is the space of absolutely bounded real-valued functions ${ }^{5}$ on $\Omega \times \mathbf{T}$, measurable on $\mathcal{F}_{t}$ for all $t \in \mathbf{T}:=\{0,1, \ldots, T\}$, the index set of consumption dates

$$
L:=L_{\infty}(\Omega)^{\mathbf{T}}=\left\{x \mid x: \Omega \times \mathbf{T} \rightarrow \mathbf{R}, x(t) \in \mathcal{F}_{t}, \text { bounded } \forall t \in \mathbf{T}\right\} .
$$

The dual of $L$ (the space of prices) is denoted $L^{*}:=L_{1}(\Omega)^{\mathbf{T}}$ as I use the Mackey topology.

The MPP enters the description of the economy in the definition of the commodity space, by making commodities be functions from the probability space. Also the information revealed by the MPP affects the commodity space because commodities at date $t \in \mathbf{T}$ have to be measurable with respect to the filtration generated by $N$ up to $t, \mathcal{F}_{t}$.

Assumption 3 There are $n<\infty$ agents indexed by $i \in I:=\{1,2, \ldots, n\}$. Each agent is described by a consumption set, $X_{i}=L_{+}$, an endowment, $w_{i} \in L_{+}$, and VonNeuman-Morgenstern preferences of the form

$$
U_{i}(x)=\sum_{t \in \mathbf{T}} \beta_{i}^{t} E_{P}\left[u_{i}(x(t))\right]
$$

where $u_{i}(x)$ is a monotone increasing, concave real-valued function. Denote the aggregate endowment by $w=\sum_{i \in I} w_{i} . w>0$ P-a.s.

\footnotetext{
${ }^{3}$ In general, $M_{k}(t)$ would be local martingales but they will be martingales for processes that admit a locally bounded intensity.

${ }^{4} \mathrm{~A}$ set of martingales forms a martingale basis if the space of all linear combinations of the martingales in the set is the space of all martingales. See Bremaud[1981] or Last and Brandt[1995] and the Appendix for more formal definitions and the corresponding martingale representation theorems.

${ }^{5}$ Naturally, I identify functions as being equal up to sets of measure zero, $P$ a.s.
} 
Note that I am assuming agents have common priors. This assumption will be important in section 4 . The assumptions on endowments, consumption sets and preferences are quite standard.

This economy is completely described by $\mathcal{E}:=\left(L,\left(U_{i}, w_{i}\right)_{i \in I}\right)$. $\mathcal{E}$ is a very simple, and well-behaved economy as we will see in the next section.

\section{Existence of Equilibrium}

This section looks at the welfare properties of the economy and establishes that a Walrasian equilibrium can be implemented as a Radner equilibrium. This is essential because it provides the link between Walrasian equilibrium and a Radner equilibrium. In particular, it will characterize security prices and trading which I need to establish the relationship between risk sharing agreements in the Walrasian equilibrium and the way agents trade in a security markets equilibrium to implement those agreements.

The discrete time economy has very well-understood properties, in particular, the two fundamental theorems of welfare economics hold and an equilibrium exists. But what is most interesting from the point of view of down-to-earth applications is the relationship between the results for the state-contingent commodity economy and the one with financial markets. I will show that any Walrasian equilibrium of the state-contingent commodity trading economy can be implemented as a security trading equilibrium. For this I adapt the result in Duffie and Huang[1985] to deal with intermediate consumption in the hybrid time framework.

\subsection{Arrow-Debreu Equilibrium}

When setting up a general modeling framework as I propose to do here, one wants to ensure that the framework has certain properties. Key properties are whether the two fundamental theorems of welfare economics hold, i.e., whether an equilibrium is Pareto optimal, whether a Pareto optimal allocation can be decentralized as an equilibrium, and whether a Walrasian equilibrium exists. These properties are generally well-understood but we will need to refer to them when analyzing the economy with financial markets.

A Walrasian equilibrium is an allocation of resources and a price for commodities such that each agent is assigned an allocation in her budget constraint such that she cannot be better off by additional trading. Such allocations are limited in that they cannot exhaust available resources. This is defined formally as 
Definition 1 An allocation, $x=\left(x_{1}, \ldots, x_{n}\right)$ is attainable if $x_{i} \in X_{i}$ for all $i \in I$ and $\sum_{i \in I} x_{i} \leq w$. Denote the set of all attainable allocations by $Z$.

With this one can define a Walrasian equilibrium

Definition 2 An allocation $x$ and a price vector $p \in L^{*}$ define a Walrasian Equilibrium if $p \cdot w \neq 0, x$ is attainable, for all $i \in I, p \cdot x_{i} \leq p \cdot w_{i}$, and $p \cdot x_{i}^{\prime}>p \cdot w_{i}$ for all $x_{i}^{\prime}$ such that $U_{i}\left(x_{i}^{\prime}\right)>U_{i}\left(x_{i}\right)$.

The two welfare theorems for the state-contingent commodity economy follow from the standard result (the first welfare theorem) and from a theorem in Bewley[1972] (the second welfare theorem) ${ }^{6}$. Also, the Bewley's theorem ensures that a Walrasian equilibrium exists.

\subsection{Radner Equilibrium}

In general, state-contingent commodity markets are not observed in the real world. The main motivation for this framework is its value for down-to-earth applications. What we do observe are markets with financial sectors that trade in securities so it is important to know whether the welfare implications of the state-contingent commodity trading economy carry over to financial markets. In particular, one would like that the Walrasian equilibrium, whose existence and welfare properties we looked at in the previous section, can be implemented as an equilibrium in security trading. The appropriate notion of equilibrium is that of Radner[1972]: an allocation together with security trades for each agent, such that the trades are feasible and they, together with the allocations, satisfy the agents dynamic budget constraints. Also, agents cannot improve their allocations by changing their trades in securities at any time given security and spot commodity prices.

\subsubsection{Security Markets}

In order to study security markets I need to define what a security is and what do I mean by trading in security markets.

Securities When one thinks of a security it is usually in the form of a stock: a claim on a firm's assets that receives dividends on a regular basis (quarterly, biannually, or annually) which depend on the firm's performance. If the firm is doing well it will pay a higher dividend, if not a lower, maybe a zero dividend. And when one thinks of trading in a financial market, the

\footnotetext{
${ }^{6}$ For more details see Mas-Collel and Zame[1991].
} 
stock market comes to mind, where these stocks (claims to future dividends) are exchanged at some mutually agreed price. The idea behind stocks can be generalized to model all kinds of financial contracts:

Definition 3 A security is a promise to deliver a fixed, possibly random, quantity of real commodities at dates $t \in \mathbf{T}$. Securities are indexed by $j \in$ $\mathcal{J}:=\{1, \ldots, J\} . d_{j}(t) \in \mathcal{F}_{t}$ is a random variable representing the dividends paid by security $j$ at date $t \in \mathbf{T}$ and $d_{j}(0)=0$.

This abstract definition is broad enough to cover all financial instruments $^{7}$ : stocks, bonds, options, futures, etc. It also covers insurance contracts, for what are insurance contracts but

an exchange of money now for money payable contingent on the occurrence of certain events. (Arrow[1984])

Hence I will refer to the securities in the context of insurance markets as insurance contracts, where an insurance contract is a claim on commodities at certain dates contingent on the occurrence of certain events.

Trading Trading of stocks takes place in stock exchanges and are described in terms of units of stocks involved in an exchange. In this framework I wish to allow agents to trade freely at any date, even at times for which no commodities are defined - in between consumption dates. This is quite standard in finance models and is usually modeled by describing stock holdings as a function of time: $\theta_{j}^{i}: \Omega \times \mathcal{T} \rightarrow \mathbf{R}$, where $\theta_{j}^{i}(t)$ is a random variable representing the number of shares of security $j$ held by agent $i$ at date $t \in \mathcal{T}:=[0, T]$ (where trading is the change in stock holdings). But describing trades just as a stochastic process is not enough to accurately characterize stock holdings:

Definition 4 The set of feasible trading strategies, $\Theta$, is the set of P-locally integrable, predictable processes on $\mathbf{F}$, such that their discounted value at any date $t$ is finite.

This defines trading as dynamic plans that imply stock holdings $(\theta \in \Theta)$ that do not anticipate future news (are predictable and measurable with respect to $\mathbf{F}$ ) and that do not create money from nothing (are $P$-locally

\footnotetext{
${ }^{7}$ Note that the securities I will be using in this paper are securities written on exogenous events. Many financial instruments traded in stock markets are written in a way that depends also on prices. Such securities can be included and be priced as redundant securities once a complete markets equilibrium has been established.
} 
integrable and have finite discounted value). The discounted value of a portfolio between consumption dates is the stochastic integral of the trading process with respect to the discounted gains process:

$$
V(s)=V(t)+\int_{t}^{s} \theta(u) d G^{*}(u), \quad t \leq s<t+1, t \in \mathbf{T},
$$

where $V(t)$ is the initial value of the portfolio at date $t$, and $G^{*}(s)$ is a stochastic process representing the discounted gains from the security (the price plus the accumulated dividends) at date $s$. The combination of allowing stock holdings to be locally integrable and have finite value in discounted terms strikes a balance between allowing agents enough flexibility to attain consumptions that may require trades bounded away from zero into the infinite horizon, while ruling out money-making strategies such as Ponzi schemes.

\subsubsection{Security Pricing}

Having defined the main elements of security markets, I wish to look at how securities would fare within the state-contingent commodity market of the previous section. This will give us an idea of how security markets and state contingent commodity markets are related, and start us on how and if a Walrasian equilibrium can be implemented as a security market equilibrium ${ }^{8}$.

The first result I need is that the state-contingent value of a security's dividends can be expressed as the expected discounted value of the dividends. This involves a change of probability measure (from $P$ to $Q$, where $Q$ is called the martingale measure). The new measure, $Q$, will turn out to be very important when it comes to proving that a Walrasian equilibrium can be implemented as a security market equilibrium. Nevertheless, this result is of interest in itself as it relates two important notions: one is the intuitive economic notion of pricing an investment today as the expected discounted value of its future returns, and the other, the price of those returns in the context of a global economy-wide equilibrium.

Theorem 1 Given any $\pi>0$, there exists a measure $Q$ and an interest rate process, $r(t), t \in \mathbf{T}$, such that

$$
p \cdot d=\sum_{t \in \mathbf{T}} E_{Q}\left[d^{*}(t)\right],
$$

\footnotetext{
${ }^{8}$ Here I am following the approach in Ellickson and Penalva[1997].
} 
where $d^{*}(t)$ denotes the discounted dividend

$$
d^{*}(t)=\frac{d(t)}{\prod_{s=1}^{t} 1+r(s)} .
$$

Proof: I will proceed by constructing $Q$ and $r$ and showing that $Q$ is a probability measure and that Equation (1) holds.

First, note that $L^{*}=L^{1}$, and hence $p \in L^{*}$ can be represented by a vector of random variables, $\pi(t) \in \mathcal{F}_{t}$ such that $E_{P}[\pi(t)]<\infty$, and for all $x \in L$,

$$
p \cdot x=\sum_{t \in \mathbf{T}} E_{P}[\pi(t) x(t)]
$$

Define the interest rate process $r(t)$ as

$$
1+r(t)=\frac{\pi(t-1)}{E_{P}\left[\pi(t) \mid \mathcal{F}_{t-1}\right]}, \quad \forall t \in\{1,2, \ldots, T\}
$$

As $\pi>0, r(t)$ is well-defined. Construct the martingale measure using the functions $\xi: \mathbf{T} \times \Omega \rightarrow \mathbf{R}, z: \mathbf{T} \times \Omega \rightarrow \mathbf{R}$

$$
\begin{aligned}
z(t, \omega) & =\frac{\pi(t, \omega)}{E_{P}\left[\pi(t) \mid \mathcal{F}_{t-1}\right]} \\
\xi(t, \omega) & =\prod_{s=1}^{t} z(s, \omega)
\end{aligned} \quad \forall t \in\{1,2, \ldots, T\}
$$

Let $\xi(0)=z(0)=1$. $\xi(t)$ defines the Radon-Nikodym derivative of an absolutely continuous measure $Q(t)$ with respect to $P$ on the filtration $\mathcal{F}_{t}$. For any event $A \in \mathcal{F}_{t}$

$$
Q(t, A)=\int_{A} \xi(t) d P=\int_{A} \prod_{s=1}^{t} \frac{\pi(s)}{E_{P}\left[\pi(s) \mid \mathcal{F}_{s-1}\right]} d P
$$

$Q(t)$ is a probability measure

$$
\begin{aligned}
Q(t, \Omega) & =\int_{\Omega} \prod_{s=1}^{t} \frac{\pi(s) d P}{E_{P}\left[\pi(s) \mid \mathcal{F}_{s-1}\right]} \\
& =E_{P}\left[\prod_{s=1}^{t} \frac{\pi(s) d P}{E_{P}\left[\pi(s) \mid \mathcal{F}_{s-1}\right]}\right] \\
& =E_{P}\left[E_{P}\left[\prod_{s=1}^{t} \frac{\pi(s) d P}{E_{P}\left[\pi(s) \mid \mathcal{F}_{s-1}\right]} \mid \mathcal{F}_{t-1}\right]\right] \\
& =E_{P}\left[\prod_{s=1}^{t-1} \frac{\pi(s) d P}{E_{P}\left[\pi(s) \mid \mathcal{F}_{s-1}\right]}\right]
\end{aligned}
$$


By repeatedly conditioning on $\mathcal{F}_{s}, s=t-2, \ldots, 1,0$, inside $E_{P}[\cdot]$, we obtain $Q(t, \Omega)=1$.

As the $Q(t)$ s are consistent, by the Kolmogorov Extension Theorem there exists a measure $Q$ such that for all $A \in \mathcal{F}_{t}, t \in \mathbf{T}: Q(A)=Q(t, A)$.

All that is left to show is that Equation (1) holds. Start with

$$
E_{P}[\pi(t) d(t)]
$$

for an arbitrary $t \in \mathbf{T}$.

$$
\begin{aligned}
E_{P}[\pi(t) d(t)]= & E_{P}\left[\prod_{s=1}^{t-1} \frac{\pi(s)}{\pi(s)} \prod_{s=1}^{t-1} \frac{E_{P}\left[\pi(s) \mid \mathcal{F}_{s-1}\right]}{E_{P}\left[\pi(s) \mid \mathcal{F}_{s-1}\right]}\left(\frac{E_{P}\left[\pi(t) \mid \mathcal{F}_{t-1}\right]}{E_{P}\left[\pi(t) \mid \mathcal{F}_{t-1}\right]}\right) \pi(t) d(t)\right] \\
= & E_{P}\left[E_{P}\left[\pi(t) \mid \mathcal{F}_{t-1}\right] \prod_{s=1}^{t-1} \frac{E_{P}\left[\pi(s) \mid \mathcal{F}_{s-1}\right]}{\pi(s)} \prod_{s=1}^{t-1} \frac{\pi(s)}{E_{P}\left[\pi(s) \mid \mathcal{F}_{s-1}\right]} \cdot\right. \\
& \left.\cdot \frac{\pi(t)}{E_{P}\left[\pi(t) \mid \mathcal{F}_{t-1}\right]} d(t)\right] \\
= & E_{P}\left[\frac{\prod_{s=1}^{t} E_{P}\left[\pi(s) \mid \mathcal{F}_{s-1}\right]}{\prod_{s=1}^{t-1} \pi(s)} \xi(t) d(t)\right]
\end{aligned}
$$

As $\pi(0)=1$,

$$
\begin{aligned}
E_{P}[\pi(t) d(t)] & =E_{P}\left[\prod_{s=1}^{t} \frac{E_{P}\left[\pi(s) \mid \mathcal{F}_{s-1}\right]}{\pi(s-1)} \xi(t) d(t)\right] \\
& =E_{P}\left[\xi(t) \prod_{s=1}^{t} \frac{1}{1+r(s)} d(t)\right] \\
& =E_{Q}\left[d^{*}(t)\right]
\end{aligned}
$$

So that

$$
p \cdot d=\sum_{t \in \mathbf{T}} E_{P}[\pi(t) d(t)]=\sum_{t \in \mathbf{T}} E_{Q}\left[d^{*}(t)\right] .
$$

\subsubsection{Implementing a Walrasian Equilibrium}

In this section I will show how a Walrasian equilibrium can be implemented as a security trading equilibrium. 
As with the Walrasian equilibrium, I need to introduce a couple of definitions to formalize two concepts: what the budget constraint is for an agent in an economy that only has security markets, and what is an equilibrium in that economy.

First, denote an agent's net trades at date $t \in \mathbf{T}$ by $\Delta_{w} x_{i}(t, \omega):=$ $\left(x_{i}(t, \omega)-w_{i}(t, \omega)\right)$.

Given an interest rate process, denote the cumulative discounted value of past dividends at date $s \in \mathcal{T}:=[0, T], s \in[t, t+1), t \in \mathbf{T}$, by $D^{*}(t)$,

$$
D^{*}(s)=\sum_{u=1}^{t} d^{*}(u)
$$

the undiscounted cumulative dividends as

$$
D(s)=\sum_{u=1}^{t} d(u),
$$

and the cumulative discounted value of future dividends as

$$
D^{*+}(s)=\sum_{u=t+1}^{T} d^{*}(u) .
$$

Define the gain process of a security with cumulative dividends $D(t)$ and security price process $S(t)$ as

$$
G(t)=S(t)+D(t) \quad \forall t \in \mathcal{T} .
$$

Definition 5 An agent's budget constraint, $B_{i}\left(S, w_{i}\right)$, is the set of consumption bundles the agent can obtain given security prices, and using feasible trades

$$
B_{i}\left(S, w_{i}\right)=\left\{\begin{array}{l|l}
x_{i} \in X_{i} & \begin{array}{l}
\exists \theta \in \Theta, \\
\sum_{s=0}^{t} \Delta_{w} x_{i}(s)+\sum_{j=0}^{J} \theta_{i}^{j}(t)\left(S_{j}(t)+d_{j}(t)\right) \\
=\sum_{j=0}^{J} \int_{0}^{t} \theta_{i}^{j}(t) d G_{j}(t), \quad \forall t \in \mathbf{T}, \quad P-\text { a.s. }
\end{array}
\end{array}\right\}
$$

With this definition I can define an equilibrium in security trading.

Definition 6 A Radner equilibrium is a triple of security prices, trading strategies, and consumption allocations, $\left(S, \theta, x^{*}\right)$, such that 
1. For all $i \in I, x_{i}^{*} \in B_{i}\left(S, w_{i}\right)$

2. For all $i \in I, U_{i}\left(x_{i}\right)>U_{i}\left(x_{i}^{*}\right)$ implies $x_{i} \notin B_{i}\left(S, w_{i}\right)$

3. $\sum_{i \in I} \theta_{i}=0$, and $x \in Z$.

Given the differences between a Radner equilibrium and a Walrasian one, it is a bit surprising that one can be transformed into the other.

Theorem 2 Given a Walrasian equilibrium pair $(p, x)$ for economy $\mathcal{E}$, there exists $K+1$ securities, and a triple $\left(S, \theta, x^{*}\right)$ such that

1. $\left(S, \theta, x^{*}\right)$ is a Radner equilibrium

2. $x^{*}=x P$-a.s.

Proof: ${ }^{9} \quad$ This proof proceeds as follows: first I will use previous results to construct a pricing scheme. This pricing scheme is constructed from Walrasian equilibrium prices, $p$. It also takes into account that trading takes place at intermediate dates, $s \in[t, t+1], t \in \mathbf{T}$. Then, I describe the properties of the 'right' kind of securities needed and construct the trading plans. Finally, I show that the prices of the dividends constructed from the announced pricing scheme, together with the trading plans and the Pareto optimal allocation form a Radner equilibrium.

The interiority of $w$ and monotonicity of preferences implies that $p>0$. Hence, by Theorem 1, there exists a martingale measure $Q$ and interest rates $r(t)$ that describe the value of a stream of dividends as the expected discounted value of those dividends.

Define the following pricing scheme: for any dividend process $(d(t))_{t \in \mathbf{T}}$, and any $s \in[t, t+1), t \in \mathbf{T}$, let the discounted value of the stream of future dividends be

$$
S^{*}(s)=E_{Q}\left[D^{*+}(t+1) \mid \mathcal{F}_{s}\right]
$$

and the undiscounted price be

$$
S(s)=S^{*}(s) \prod_{u=1}^{t}(1+r(u)),
$$

where for $s \in[0,1), S(s)=S^{*}(s)$. Note that the discounted gains process, $G^{*}(t)=S^{*}(t)+D^{*}(t)$ is a martingale. For any $t \in \mathbf{T}, s \in[t-1, t)$

\footnotetext{
${ }^{9}$ This proof is a modification of the proof of similar results in Duffie and Huang[1985] and Duffie and Zame[1989].
} 


$$
\begin{aligned}
E_{Q}\left[S^{*}(t)+D^{*}(t) \mid \mathcal{F}_{s}\right] & =E_{Q}\left[E_{Q}\left[\sum_{u=t+1}^{T} d^{*}(u) \mid \mathcal{F}_{s}\right]+\sum_{u=\mathbf{0}}^{t} d^{*}(u) \mid \mathcal{F}_{s}\right] \\
& =E_{Q}\left[\sum_{u=t+1}^{T} d^{*}(u) \mid \mathcal{F}_{s}\right]+E_{Q}\left[d^{*}(t) \mid \mathcal{F}_{s}\right]+\sum_{u=\mathbf{0}}^{t-1} d^{*}(u) \\
& =S^{*}(s)+D^{*}(s)
\end{aligned}
$$

In order to be able to decentralize the Pareto optimal allocation, I need to have a set of securities such that trading in them will be sufficient to attain the desired allocation. Following method of Duffie and Huang[1985], construct a sequence of riskless bonds and $K$ securities as follows:

1. Each riskless bond exists for a single period: at each date $t \in \mathbf{T}$ a new bond is created which pays $1+r(t+1)$ units of consumption at date $t+1$ and is valueless thereafter. Its price $S_{0}(s)$ at any $s \in[t, t+1)$ is 1 .

2. The gains processes of the other $K$ securities, $G_{j}^{*}(t)$, form a basis for the space of $Q$-martingales.

Note that I have given the exact number of securities needed. This is because the space of martingales defined by a marked point process with $K$ marks has martingale multiplicity $K$ which guarantees that such securities exist (see the Appendix).

Given these securities and their prices, define the $Q$-martingale $X_{i}$ for all $i \in I \backslash\{1\}$ as

$$
X_{i}(t)=E_{Q}\left[\sum_{s=0}^{T} \Delta_{w} x_{i}^{*}(s) \mid \mathcal{F}_{s}\right] \text {, }
$$

where

$$
\Delta_{w} x_{i}^{*}(t)=\frac{\Delta_{w} x_{i}(t)}{\prod_{s=1}^{t} 1+r(s)},
$$

and the $x_{i}$ 's are agent $i$ 's Pareto optimal allocation from the statement of the theorem.

By the martingale representation theorem ${ }^{10}$ there exists $\theta_{j}^{i} \in \Theta$ such that

$$
X_{i}(t)=\sum_{j=1}^{K} \int_{0}^{t} \theta_{j}^{i}(s) d G_{j}^{*}(s) .
$$

\footnotetext{
${ }^{10}$ See Appendix.
} 
A great benefit of using MPPs is that these $\theta_{i}$ s are the solutions to a system of stochastic differential equations which can be used using the standard Stieltjes-Lebesgue calculus. The $\theta^{i}$ s solve

$$
\begin{array}{r}
\sum_{k \in \mathbf{K}}\left(g_{k}^{i}(t) \Delta N_{k}(t)-g_{k}^{i}(s) \lambda_{k}(s) d s\right)= \\
\sum_{j=1}^{J} \sum_{k \in \mathbf{K}}\left(\theta_{j}^{i}(s) f_{k}^{j}(s) \Delta N_{k}(s)-\theta_{j}^{i}(s) f_{k}^{j}(s) \lambda_{k}(s) d s\right)
\end{array}
$$

where $\Delta N_{k}(s)=N_{k}(s+d s)-N_{k}(s)$, and $g_{k}^{i}(s)$ and the $f_{k}^{j}$ s are the representations of $X_{i}(t)$ and the discounted security gains processes.

Let those $\theta_{j}^{i}$ be the trading plans for all but the first agent, $i \in I$. In order for these trading strategies to be budget feasible, define the agents' trade in the riskless bond, $\theta_{0}^{i}$, as follows: for all $s \in[t, t+1), t \in \mathbf{T}$,

$$
\theta_{0}^{i}(s)=X(s)-E_{Q}\left[\sum_{u=0}^{t} \Delta_{w} x_{i}^{*}(u)\right]-\sum_{j=1}^{K} \theta_{j}^{i}(s) G_{j}^{*}(s) .
$$

This ensures that the agents' plans will be budget feasible by construction (note that $d G_{0}^{*}(s)=0$ for all $s \in \mathcal{T}$, the change in the gains from the bond is always zero). To show $\theta_{0}^{i}(s) \in \Theta$, I refer to Lemma A.1 in Duffie and Huang[1985]. The outline of the proof is that by the left limit property of martingales:

$$
\begin{aligned}
& \sum_{j=1}^{K} \int_{0}^{t} \theta_{j}^{i}(s) d G_{j}^{*}(s)-\sum_{j=1}^{K} \theta_{j}^{i}(t) G_{j}^{*}(t) \\
& =\sum_{j=1}^{K} \int_{0}^{t-} \theta_{j}^{i}(s) d G_{j}^{*}(s)-\sum_{j=1}^{K} \theta_{j}^{i}(t) G_{j}^{*}(t)+\theta_{j}^{i}(s) \Delta G_{j}^{*}(t)
\end{aligned}
$$

Using,

$$
\theta_{j}^{i}(t) G_{j}^{*}(t)=\theta_{j}^{i}(t) G_{j}^{*}(t-)+\theta_{j}^{i}(s) \Delta G_{j}^{*}(t),
$$

demonstrates $\theta_{0}^{i}$ is predictable. $\theta_{0}^{i}$ is now obviously locally integrable and has finite discounted value.

For the first agent, let

$$
\theta^{1}=-\sum_{i=2}^{n} \theta^{i}
$$

$\theta^{1} \in \Theta$ because $\Theta$ is closed under finite sums. 
I claim that $\left(\left(S_{j}\right)_{j=\mathbf{0}}^{K},\left(\theta^{i}, x_{i}\right)_{i \in I}\right)$ is a Radner equilibrium for $\mathcal{E}$. By construction, $x_{i}$ is agent $i$ 's optimal allocation from the Walrasian equilibrium for all $i \in I . x_{1}$ is also agent one's optimal allocation from the statement of the theorem. This follows from the fact that $\sum_{i} \theta^{i}=0$, the budget equalities for all $i \in I \backslash\{1\}$, and monotonicity of preferences.

I need to show that $x_{i}$ will also be optimal for the sequence of Radner budget constraints. To show that this allocation together with the given trades is optimal one makes use of the Pareto optimality of $x$, the definition of feasible trading strategies, and the derivation of $Q$ from equilibrium prices: if there is a feasible trading pattern $\theta \in \Theta$ such that for all $i \in I, U_{i}\left(x_{i}^{\prime}\right) \geq$ $U_{i}\left(x_{i}\right)$, and there is some agent $j$ such that $U_{j}\left(x_{j}^{\prime}\right)>U_{j}\left(x_{j}\right)$ then it will be more expensive, $p \cdot x_{j}^{\prime}>p \cdot x_{j}$, and any feasible trading strategy implementing it will have the property that

$$
\sum_{t \in \mathbf{T}} E_{Q}\left[\frac{x_{j}^{\prime}(t)}{\prod_{s=1}^{t} 1+r(s)}\right]>\sum_{t \in \mathbf{T}} E_{Q}\left[\frac{x_{j}(t)}{\prod_{s=1}^{t} 1+r(s)}\right] .
$$

From the budget constraint at date zero,

$$
\sum_{t \in \mathbf{T}} E_{Q}\left[\frac{x_{j}(t)}{\prod_{s=1}^{t} 1+r(s)}\right]=\sum_{t \in \mathbf{T}} E_{Q}\left[\frac{w_{j}(t)}{\prod_{s=1}^{t} 1+r(s)}\right] .
$$

Substituting this into the previous equation we obtain

$$
\sum_{t \in \mathbf{T}} E_{Q}\left[\frac{x_{j}^{\prime}(t)}{\prod_{s=1}^{t} 1+r(s)}\right]>\sum_{t \in \mathbf{T}} E_{Q}\left[\frac{w_{j}(t)}{\prod_{s=1}^{t} 1+r(s)}\right],
$$

But this last line contradicts $x^{\prime}$ being budget feasible at date zero.

\section{Risk Sharing and Trading Patterns}

In this section I will show that the risk-averse agents in $\mathcal{E}$ would optimally wish to pool their risks and look at what this risk sharing implies about the way agents behave in security markets. Risk pooling is a standard and intuitive property of the contingent commodity equilibrium. The main questions I wish to answer is how this risk-sharing rule is implemented in a security trading equilibrium and whether agents' trading patterns will look anything like observed trading patterns. The main result of this paper is that this model predicts agents will optimally share risks by buying insurance on 
themselves and participating in others' risks by buying securities that pool those risks: derivatives writhen on the aggregate endowment process such as mutual funds, catastrophe bonds, etc. In order to characterize the portfolio of securities traded, I introduce the concept of a risk class, where a risk class describes a set of shocks that can be pooled as a single one in terms of their effect on the aggregate endowment. I also describe how agents' idiosyncratic risks have to be related to the risk class, the pool, so that agents would only need to trade on one personal insurance contract per risk class. Putting these two together I obtain a full characterization of the agents' trading portfolios.

\subsection{Risk Sharing}

The first step is to look at how agents would behave if markets for all commodities were available. As we saw in the second section, in such a circumstance agents will attain first best Pareto optimal allocations. In this section I wish to characterize these first best allocations to see if they could be attained when the economy uses financial markets rather than statecontingent commodity markets.

It will be useful to make an additional assumption. I will assume that endowments ignore the exact timing of the jumps. Define the filtration on T:

$$
\mathcal{G}_{t}=\sigma(N(s), s \leq t, s \in \mathbf{T}) \quad t \in \mathbf{T} .
$$

Assumption 4 For all $i \in I, w_{i}(t)$ is measurable with respect to $\mathcal{G}_{t}$ for all $t \in \mathbf{T}:=\{0,1, \ldots, T\}$, the index set of consumption dates.

Let $\mathcal{E}^{\prime}$ represent the economy $\mathcal{E}$ but with endowments described by Assumption 4 . The main consequence of this assumption is that $\mathcal{G}_{t}$ can be described by a countable partition, $G_{t}:=\left\{G_{t}^{1}, G_{t}^{2}, \ldots\right\}$, so that consumption at date $t$ can be represented by $x(t)=\left(x\left(G_{t}^{1}\right), x\left(G_{t}^{2}\right), \ldots\right)$, where $x\left(G_{t}^{j}\right)$ is a constant for all $j$ (a similar representation holds for endowments and prices). With this one can show that agents will wish to pool their risks in equilibrium.

Theorem $3{ }^{11}$ There exists a Walrasian equilibrium $(p, x)$ for $\mathcal{E}^{\prime}$ such that the Walrasian equilibrium price, $p \in L^{*}$, can be represented by $g(t, w)$ where

\footnotetext{
${ }^{11}$ This result is a corollary of Huang[1987]'s representative agent construction itself an extension of earlier results by Wilson[1968], and Diamond[1967].
} 
for any $x \in L$

$$
p \cdot x=\sum_{t \in \mathbf{T}} E_{P}[g(t, w) x(t)]
$$

and the corresponding equilibrium allocations, $\left(x_{i}^{*}\right)_{i \in I}$ can be represented by the sequence of vectors

$$
x_{i}^{*}=\left(f_{i}(t, w)\right)_{t \in \mathbf{T}} .
$$

Proof: First note that an equilibrium will exist (Bewley[1972]) so all I need to show is that equilibrium consumptions and prices will have the desired property.

Let us look at consumption and prices for an equilibrium at each consumption date separately. First note that the price functional $p$ can be represented by the sequence of random vectors, $\pi(t)$, such that

$$
p \cdot x=\sum_{t=0}^{T} E_{P}[\pi(t) x(t)] .
$$

Given that $\mathcal{G}_{t}$, can be described by a partition, take the consumption allocation for an arbitrary agent, $i$, at date $t$ implied by the allocation $x, x_{i}(t)$, and two disjoint events $G_{t}^{k}, G_{t}^{l} \in G_{t}, k \neq l$, with the same aggregate endowment, $w\left(t, G_{t}^{k}\right)=w\left(t, G_{t}^{l}\right)$, and with positive probabilities, $P\left(G_{t}^{k}\right), P\left(G_{t}^{l}\right)>0$. I want to show that for any agent, $i \in I$, $x_{i}\left(t, G_{t}^{k}\right)=x_{i}\left(t, G_{t}^{l}\right)$. The first-order conditions for agents $i$ and $j$ at date $t \in \mathbf{T}$ imply:

$$
\frac{P\left(G_{t}^{k}\right) \pi\left(t, G_{t}^{k}\right)}{P\left(G_{t}^{l}\right) \pi\left(t, G_{t}^{l}\right)}=\frac{P\left(G_{t}^{k}\right) u_{i}^{\prime}\left(x_{i}\left(t, G_{t}^{k}\right)\right)}{P\left(G_{t}^{l}\right) u_{i}^{\prime}\left(x_{i}\left(t, G_{t}^{l}\right)\right)}=\frac{P\left(G_{t}^{k}\right) u_{j}^{\prime}\left(x_{j}\left(t, G_{t}^{k}\right)\right)}{P\left(G_{t}^{l}\right) u_{j}^{\prime}\left(x_{j}\left(t, G_{t}^{l}\right)\right)}
$$

Note that the rate of time preference, $\beta_{i}$, cancels from the top and the bottom for both agents. From strict concavity: $u_{i}^{\prime}\left(x_{i}\left(t, G_{t}^{k}\right)\right) / u_{i}^{\prime}\left(x_{i}\left(t, G_{t}^{l}\right)\right)=1$ if and only if $x_{i}\left(t, G_{t}^{k}\right)=x_{i}\left(t, G_{t}^{l}\right)$ (and similarly for agent $j$ ), so that this condition together with the previous equation imply that if $x_{i}\left(t, G_{t}^{k}\right)>x_{i}\left(t, G_{t}^{l}\right)$, it will also be the case for $j$ (and all other agents). By monotonicity of preferences, this would lead to an allocation that is not feasible (as $\left.w\left(t, G_{t}^{k}\right)=w\left(t, G_{t}^{l}\right)\right)$, so that the only feasible allocation that satisfies the first-order conditions is $x_{i}\left(t, G_{t}^{k}\right)=x_{j}\left(T, G_{t}^{l}\right)$ for all agents. As for the representation of prices: $x_{i}\left(t, G_{t}^{k}\right)=x_{i}\left(t, G_{t}^{l}\right)$, implies $\pi\left(t, G_{t}^{k}\right) / \pi\left(t, G_{t}^{l}\right)=1$. The existence of the functions, $g_{i}$ and $f$, is now merely an issue of representation. 
What I have shown is that agents' optimal allocations will ignore what happens to each agent's endowment and give each agent a share of the aggregate endowment. Note that this risk sharing agreements are the generalization of insurance to situations with aggregate endowment risk. In such cases, it is physically impossible to provide 'full insurance' to every agent and eliminate uncertainty completely. If there is aggregate uncertainty, agents will participate in the aggregate risk and they will do so in an optimal way: letting the less risk-averse agents carry a higher proportion of the risk. Ideally, this is what insurance contracts should do, and we see some of this with coinsurance and partial insurance, specially at the level of major economic enterprises such as trade (the risk stemming from currency fluctuations) where insurance takes the form of hedging.

\subsection{Trading Patterns}

Risk pooling is interpreted in financial markets as insurance and hedging. The aim of this section is to make this link explicit and see how risk pooling is translated into optimal trading strategies that have the interpretation of taking insurance and hedging.

Let us look at the earthquake example: suppose that you have an economy in which all agents have fixed but when an earthquake happens the economy loses a fixed amount $L$. The risk sharing result from the previous section tells us that for purposes of optimally allocating consumption it does not matter who was hit: whether the earthquake destroyed two hundred homes in Bel Air where each house costs one million dollars or whether it destroyed two thousand homes in Inglewood each costing one hundred thousand dollars. In a sense, agents consume as if they had full insurance conditional on the number of earthquakes.

The question I want to address is how would this optimal allocation be implemented. In a small isolated and relatively homogenous economy, such as a rural village in India or in medieval Europe, one can imagine that it would relatively straight-forward to set up institutions that would enforce such outcomes. On the other hand, as the economy develops and becomes larger and more complex, the kind of institutions that enforced risk sharing in small isolated communities would not function well. What one can observe is that such economies tend to develop financial and insurance markets to share risks. How would an insurance market deal with this earthquake risk? If one is to judge by the experience in California, insurance companies will claim that they are not willing to be exposed to such risks and would demand the government to put up a reserve fund to help mitigate their 
exposure, and the government would agree. This suggests that insurance markets are somehow unable to deal with this risk. But, looking back to the risk sharing optimal allocations, it seems that there is no reason for the government and insurance companies to bear all this risk. Agents would optimally participate in this risk as long as the idiosyncratic component (fluctuations in their private endowments) was taken care off.

So let us look at how this intuition can be formalized within the model I am using. First, we have a result about risk sharing in a Walrasian equilibrium, Theorem 3, which establishes the property of optimal risk sharing, $x_{i}(t)=f_{i}(w(t), t)$, and tells us something about state-contingent commodity prices, $\pi(t)=g(w(t), t)$. Then, we also have a result on how a Walrasian equilibrium can be implemented as a Radner equilibrium so that the optimal allocations with security trading will be the same as those from the Walrasian equilibrium. A key part of the proof of that result is that the value of the agent's net trades, $X_{i}(t)$, is given by the $Q$-martingale

$$
\begin{aligned}
X_{i}(t) & =E_{Q}\left[\sum_{s=\mathbf{0}}^{T} \Delta_{w} x_{i}^{*}(s) \mid \mathcal{F}_{t}\right] \\
& =\sum_{s=\mathbf{0}}^{T} E_{P}\left[\frac{\pi(s)}{\pi(0)}\left(x_{i}(s)-w_{i}(s)\right) \mid \mathcal{F}_{t}\right] .
\end{aligned}
$$

and that this martingale can be represented as a linear combination of other martingales. The weights in this linear combination represent stock holdings and the other martingales are the cumulative discounted gains of some existing securities. Putting these two results together we obtain

$$
X_{i}(t)=\sum_{s=0}^{T} E_{P}\left[\frac{g(w(s), s)}{g(w(0), 0)}\left(f_{i}(w(s), s)-w_{i}(s)\right) \mid \mathcal{F}_{t}\right]
$$

So the key is to realize that agents only care about two things: how shocks affect them directly by changing their endowments, and how those shocks affect them indirectly by altering the aggregate endowment and prices. The way agents will deal with these effects through trades is reflected in the martingales needed to represent $X_{i}(t)$ in equation (4). From staring at this equation it seems that all that is needed is to have some securities that take care of the aggregate endowment part and some that take care of the agent's endowment. The question then remains as to what these securities are and how many does one need. 
In the particular earthquake example I am using, there seems to be no special reason why the economic consequences of leveling two hundred houses in Bel Air should be the same than leveling two thousand homes in Inglewood. But, the fact that all earthquakes have the same consequences on the aggregate endowment seems to suggest that one could take care of the effect of earthquakes on the aggregate endowment by buying a single security that pools all the individual earthquakes - those that hit Bel Air and those that hit Inglewood. This pooling idea can be generalized to all shocks that affect the aggregate endowment in the same way, and for which I define the concept of a risk class. Two types of shocks (represented by the marks $k$ and $j$ ) belong in the same risk class if one can tell what the aggregate endowment will be just be knowing the sum of the number of shocks of these two types that have taken place and not how many of jumps of each type, $k$ and $j$, have occurred.

First let me introduce a bit of notation. From above, $N_{k}(t)$, denotes the number of jumps of type $k$ that have taken place up to time $t$. I want to generalize this by allowing $N$ to be subscripted by an index set, $C$, such that $N_{C}(t)$ denotes the number of jumps of types indexed by $C$

$$
N_{C}(t)=\sum_{k \in C} N_{k}(t)
$$

I will also need the following technical result

Lemma 1 If $N$ is a $K$-marked point process, that admits intensities

$$
\lambda=\left(\lambda_{1}, \ldots, \lambda_{K}\right)
$$

then

$$
\tilde{N}(t)=\sum_{k=1}^{K} N_{k}(t),
$$

is a univariate point process with intensity

$$
\tilde{\lambda}(t)=\sum_{k=1}^{K} \lambda_{k}(t) .
$$

Proof: See Bremaud[1981], p.34.

This result ensures that $N_{C}(t)$ is a univariate point process as long as $C$ represents indeces of the original marked point process $N$. 
Assumption 5 The marks of the underlying point process $N,\{1,2, \ldots, K\}$, can be partitioned into $m$ disjoint, subsets, $C_{1}, C_{2}, \ldots, C_{m}$, such that the aggregate endowment, $w(t)$, is measurable with respect to the filtration generated by the univariate point processes, $N_{C_{j}}(t), j=1, \ldots, m$ :

$w(t) \in \mathcal{F}^{w}(t):=\sigma\left(N_{C_{1}}(s), N_{C_{2}}(s), \ldots, N_{C_{m}}(s) ; s \leq t\right) \quad \forall t \in\{0,1, \ldots, T\}$.

Definition 7 If $i, k \in C_{j}$ for any $j=1, \ldots, m$, then we say that $N_{i}$ and $N_{j}$ are in the same risk class.

Remark I The marked point process of economy $\mathcal{E}$ is described by $m$ risk classes, where $m \leq K$. This follows from the observation that in a worst case scenario, each mark can be a class of its own.

Now let us look at fluctuations in the agent's private endowment, $w_{i}$. One can approach this problem in the same way as I did the fluctuations in the aggregate endowment and define accident classes for agent $i$. I do not wish to follow that path because it implies no relationship between fluctuations in the aggregate endowment and in the agent's private endowment, while in fact fluctuations in the aggregate endowment are due to fluctuations in agent's private endowments - the loss of $L$ due to an earthquake is decomposed into $L_{i}$ 's spread over the population, agents losing their homes, forgone income, etc. In order to retain this link between private and aggregate endowment fluctuations, I introduce the following assumption

Assumption 6 For every agent, $i \in I$, and every risk class, $C_{j}$, the marks of the jumps in $C_{j}$ can be partitioned into two subsets, $A_{i}\left(C_{j}\right)$ and $C \backslash A_{i}\left(C_{j}\right)$, such that the univariate point processes

$$
N_{A_{i}\left(C_{j}\right)}=\sum_{k \in A_{i}\left(C_{j}\right)} N_{k}(t)
$$

have the property that the agent's endowment is measurable with respect to the filtration they generate

$$
\begin{gathered}
w_{i}(t) \in \mathcal{F}^{i}(t):=\sigma\left(N_{A_{i}\left(C_{1}\right)}(s), N_{A_{i}\left(C_{2}\right)}(s), \ldots, N_{A_{i}\left(C_{m}\right)}(s) ; s \leq t\right), \\
\forall t \in\{0,1, \ldots, T\} .
\end{gathered}
$$

What I intend with this assumption is to say that agent's endowments are associated with a single type of private shock for every aggregate shock (risk class). This private shock need not take place every time there is an aggregate shock in the same risk class, so the agent distinguishes the 
earthquakes that affect him and those that do not, i.e., every time houses in Bel Air are destroyed, Bruce Willis' house may or may not be destroyed, but if it is it is totally destroyed. What this implies is that agent $i$ (Bruce Willis) does not care who else was affected by the earthquake just whether there was one, and whether it destroyed his house or not.

Note that shocks need not be negative. In the same way I describe shocks as accidents (earthquakes, etc) I could also describe them as discoveries or announcements of good news, such a plentiful future harvest. The definition of risk classes and the results that follow below all go through with either interpretation. The content of the information represented by the marks is arbitrary.

Now the characterization of trading:

Theorem 4 Let the uncertainty in economy $\mathcal{E}^{\prime}$ be described by $m$ risk classes, then given a Walrasian equilibrium and any complete markets Radner equilibrium that implements it, every agent i's trades can be described by a portfolio that contains at most $m$ personalized insurance contracts, $m$ derivatives on the aggregate endowment and $a$ bond.

Proof: As we have seen, given a complete markets Radner equilibrium that implements a Walrasian equilibrium, what I need to do is construct a portfolio of securities for every agent that represent $X_{i}(t)$ in Equation (4).

But first, I want to define what the securities will be that these agents will trade in. The filtration $\mathcal{F}^{w}(t)$ has martingale multiplicity of at most $m$. Hence there are (at most) $m$ securities whose cumulative discounted gains processes form a basis for the space of $Q$-martingales with respect to $\mathcal{F}^{w}$. These securities must be measurable with respect to $\mathcal{F}^{w}$, so they can be described as derivatives on the aggregate endowment. Denote the price process of such securities at date $t$ by $S_{C_{j}}^{w}(t)$ and their dividends by $d_{C_{j}}^{w}(t)$.

Now for all $i \in I, X_{i}(t)$ is made up of prices, the agent's consumption and the agent's private endowment. Both the prices and the agent's consumption are functions of the aggregate endowment and hence measurable with respect to $\mathcal{F}^{w}$. Also, the agent's endowment is measurable with respect to $\mathcal{F}^{i}$. Hence, $X_{i}(t)$ is measurable with respect to $\mathcal{F}^{w} \cup \mathcal{F}^{i}$. $\mathcal{F}^{i}$ has martingale multiplicity of at most $m$, so, like $\mathcal{F}^{w}$, there are at most $m$ securities that form a basis for the space of $Q$-martingales with respect to $\mathcal{F}^{i}$. These securities have to be measurable with respect to $\mathcal{F}^{i}$ so they can be described as personalized insurance contracts for $i$. Denote the gains processes of such securities at date $t$ by $S_{C_{j}}^{i}$ and their dividends by $d_{C_{j}}^{i}$.

Hence, $X_{i}(t)$ is in the span of the (at most) $m$ personalized insurance 
contracts and (at most) $m$ derivative securities on the aggregate endowment. As the $X_{i}(t)$ is the same as in the original Radner equilibrium, one can use the same holdings of the riskless bond as before. So the agents' optimal portfolio can be fully described by holdings of at most one personalized insurance contract on himself and one common security per accident class, and a riskless bond, where the $m$ common securities are derivatives on the aggregate endowment such as mutual funds, catastrophe bonds, etc. At date $t$, the value of agent $i$ 's security holdings

$$
\left(\left(\theta_{C_{j}^{w}}^{i}(t), \theta_{C_{j}^{i}}^{i}(t)\right)_{j=1}^{m}, \theta_{0}^{i}(t)\right),
$$

will be

$$
\sum_{j=1}^{m}\left[\theta_{C_{j}^{w}}^{i}(t)\left(S_{C_{j}}^{w}(t)+d_{C_{j}}^{w}(t)\right)+\theta_{C_{j}^{i}}^{i}(t)\left(S_{C_{j}}^{i}(t)+d_{C_{j}}^{i}(t)\right)\right]+\theta_{0}^{i}(t)
$$

Remark II Also, note that if all the accidents in a risk class affect the agent in the same way, i.e. if $A_{i}(C)=C$ or $A_{i}(C)=\emptyset$, then the agent will only need to trade in one security to deal with that risk - be it a mutual fund or an insurance contract.

Note that, as in CAPM, one could not have a Radner equilibrium with the securities implied by the portfolio holdings in this theorem (the insurance contracts and the mutual funds) because no agent is holding the personalized insurance contracts other than the agent on which they are written. Security holdings would then not add up to zero. If one was to count how many securities are being traded one may get more than $K$ securities. What this result is saying is that every agent may be selling a different kind of insurance contract, but they will not be trading directly on each other's insurance contracts; Bruce Willis will not be selling insurance on Arnold Schwarzenegger's house (or on any house in Inglewood for that matter) but will just buy insurance on his own home and an aggregate security on the pool of everybody's risk such as a catastrophe bond. Each agent provides coverage for the others only as part of a pool, and each agent obtains coverage from that pool. This insight is key to understand why insurance and reinsurance companies exist and what their role is: eliminate the agent's idiosyncratic risk by pooling lots of them together. A similar interpretation applies to mutual fund companies, whose role in this model is to purchase individual stock holdings and sell them as a pool to individual investors. 


\section{Conclusion}

The main result in this paper illustrates the link between risk pooling in consumption as an optimal state-contingent commodity equilibrium, and insurance and hedging as the optimal trading strategies in a financial market equilibrium. I have looked at how risk-averse agents would use financial markets to share risk. I have shown that agents' trades can be described by portfolios that include a riskless bond, a common set of derivatives (written on the aggregate endowment), and personalized insurance contracts.

The link between risk pooling in consumption and security trading behaviour requires the classification of idiosyncratic shocks into groups or "risk classes'. Each of these risk classes represents a different shock on the aggregate endowment and must be treated separately from the others. But within each risk class, these idiosyncratic shocks are all equivalent and are independent of the wealth of the individual, their preferences, etc. The symmetry imposed accross agents in previous models that have studied idiosyncratic and aggregate shocks is not a necessary requirement for a parsimonious financial market. Hence one would be more willing to accept the validity of the complete markets assumption. 


\section{References}

[1] Aase, K. [1993]. "Continuous Trading in an Exchange Economy under Discontinuous Dynamics: A Resolution of the Equity Premium Puzzle." Scandinavian Journal of Management 9:Suppl. S3-S28.

[2] Arrow, Kenneth J. [1965]. "Aspects of the Theory of Risk-Bearing, lecture 3," reprinted in Collected Papers of Kenneth J. Arrow, Vol V [1984] 77-86.

[3] Bewley, T. [1972]. "Existence of Equilibria in Economies with Infinitely Many Commodities," Journal of Economic Theory 43 514-540.

[4] Breeden, D. [1979]. "An Intertemporal Asset Pricing Model with Stochastic Consumption and Investment Opportunities." Journal of Financial Economics. 7 265-296.

[5] Bremaud, P. [1981]. Point Processes and Queues: Martingale Dynamics. Springer-Verlag. New York.

[6] Cox, J. and S. Ross [1976]. "The Valuation of Options for Alternative Stochastic Processes." Journal of Financial Economics 3: 145-166

[7] Diamond, Peter [1967]. "The Role of a Stock Market in a General Equilibrium Model with Technological Uncertainty," American Economic Review 57 759-776.

[8] Duffie, D. and C. Huang [1985] "Implementing Arrow-Debreu equilibria by continuous trading of few long-lived securities." Econometrica 53: $1337-1356$

[9] Duffie, D. and W.R. Zame [1989] "The Consumption-Based Capital Asset Pricing Model." Econometrica 57: 1279-1297

[10] Ellickson, B., and J. Penalva Zuasti [1997]. "Intertemporal insurance," Journal of Risk and Insurance 64(4): 579-598.

[11] Huang, Chi-fu [1987] "An Intertemporal General Equilibrium Asset Pricing Model: The Case of Diffusion Information." Econometrica 55(1): 117-142.

[12] Last, G. and A. Brandt [1995]. Marked Point Processes on the Real Line: The Dynamic Approach. Springer-Verlag. New York. 
[13] Mace, Barbara [1991]. "Full Insurance in the Presence of Aggregate Uncertainty," Journal of Political Economy 99 928-956.

[14] Markowitz, H. [1952]. "Portfolio Selection," Journal of Finance 7 18511872.

[15] Mas-Collel, A. and W. Zame [1991]. "Equilibrium theory in infinite dimensional spaces." in Handbook of Mathematical Economics. Volume $I V$. (Hildenbrand, W., and H. Sonnenschein, ed.). Elsevier Science Publishers. B.V.

[16] Merton, Robert C. [1990] Continuous-Time Finance. Blackwell Publishers. Cambridge, Massachusetts.

[17] Penalva Zuasti, Jose S. [1997] The Theory of Financial Insurance with an Application to Earthquakes and Catastrophe Bonds. PhD Thesis, UCLA.

[18] Radner, R. [1972]. "Existence of equilibrium of plans, prices and price expectations in a sequence of markets." Econometrica 40: 1365-1379.

[19] Townsend, Robert M. [1993]. The Medieval Village Economy. Princeton University Press. Princeton, New Jersey.

[20] Townsend, Robert M. [1994]. "Risk and Insurance in Village India," Econometrica 62 539-591.

[21] Wilson, Robert [1968]. "The Theory of Syndicates," Econometrica 36 119-132. 


\section{Appendix}

\subsection{The Canonical Basis}

A special property of the canonical martingales, $M_{k}$, is that they form a basis for the space of $P$-martingales ${ }^{12}, \mathcal{M}$. It also establishes the minimum number of securities needed to span the space, as every other bases for $\mathcal{M}$ will have the same number, $K$, of orthogonal elements. This can be shown as follows:

Definition 8 Two martingales, $M$ and $N$ are orthogonal if their product $M N=\left\{M_{t} N_{t}\right\}$ is a martingale with mean 0 .

Also, for any process, $X$, let $\Delta X(t)$ denote the jump of the process at date $t$.

Lemma 2 (Elliott 1991, 9.25) For $M$ a martingale of integrable variation and any bounded corlol ${ }^{13}$ martingale $N$

$$
E[M(T) N(T)]=E\left[\sum_{s \geq \mathbf{0}} \Delta M(s) \Delta N(s)\right]
$$

The lemma describes the expected value of the product of two processes as the expected value of the sum of the product of their jumps. Naturally, a martingale of integrable variation and a bounded corlol martingale both have at most a countable number of jumps, hence the use the summation sign rather than an integral. Nevertheless note that the jump times will be random, hence the use of the index $s \geq 0$ for notational convenience. This lemma was constructed for $T=\infty$ and it naturally applies if $T<\infty$.

Theorem $\mathbf{5}$ The canonical martingales for $\mathbf{K}$ finite

$$
M_{k}(t)=N_{k}(t)-\int_{0}^{t} \lambda_{k}(s) d s
$$

$k \in \mathbf{K}$ form a martingale basis under $P$, so that $\mathcal{M}$ has dimension $K$.

\footnotetext{
${ }^{12}$ Note that the space of square-integrable martingales, $\mathcal{M}^{2}$, with the norm $E\left[A^{*}(T)^{2}\right]<$ $\infty$, where $A^{*}(t)=\sup _{s<t}|A(s)|$, and $A(t)$ is an adapted process, can be identified with the Banach space $L_{2}(\Omega, \mathcal{F}, P)$ by the map that associates the martingale $A(t) \in \mathcal{M}^{2}$ to its terminal value $A(T) \in L_{2}$ (Elliott [1982], Remark 9.8). This is the key property exploited by DH in their 1985 paper to guarantee and construct their implementation of an Arrow-Debreu equilibrium as a security market equilibrium.

${ }^{13}$ corlol := 'cadlag' - continuous on the right, left limit.
} 
Proof Define

$$
M(T)=\lim _{t \uparrow T} M(t),
$$

For all $k \in \mathbf{K}$, the set $\left\{\Delta N_{k}(t)>0, \Delta N_{j}(t)>0, j \neq k\right\}$ has probability 0 , i. e., $N_{k}$ does not charge a common jump time with any $N_{j}, j \in \mathbf{K}, j \neq k$, by definition, so that

$$
E\left[N_{j}(T) N_{k}(T)\right]=0,
$$

So that applying Elliott's lemma on any two martingales, $M_{k}, M_{j}, i \neq j$ in $\left(M_{1}, \ldots, M_{K}\right)$ we obtain

$$
\begin{aligned}
E\left[M_{k}(T) M_{j}(T)\right] & =E\left[\sum_{s \geq 0} \Delta M_{k}(s) \Delta M_{j}(s)\right] \\
& =0
\end{aligned}
$$

So that $M_{k}$ is orthogonal to every other canonical martingale, $M_{j}$. As the index $k$ is arbitrary, the vector of martingales $\left(M_{1}, \ldots, M_{K}\right)$ consists of orthogonal martingales. As $\mathbf{F}$ is the filtration generated by $N:=\left(N_{1}, \ldots, N_{K}\right)$, $\left(M_{1}, \ldots, M_{K}\right)$ form a martingale basis for $\mathcal{M}$. As all martingale basis have the same dimension, the dimension of $\mathcal{M}$ is $K$.

\subsection{Martingale Representation}

There is a second property associated with the canonical martingales which sets marked point processes apart from continuous processes such as Brownian Motion. This has to do with the way the canonical martingales can be used to represent all adapted, right-continuous processes, of which martingales is a subset.

Theorem $6{ }^{14}$ Let $\{G(t): t \geq 0\}$ be an adapted and right-continuous process. Assume the existence of a sequence $t_{n}, n \in N$, of stopping times satisfying

$$
t_{n} \uparrow \infty \quad Q-\text { a.s. }
$$

such that $\left\{G\left(t \wedge t_{n}\right): t \geq 0\right\}$ is for all $n \in N$ a uniformly integrable martingale. Then there exists a predictable vector $f$ such that

$$
\sum_{k \in \mathbf{K}} \int_{0}^{t}\left|f_{k}(s)\right| \lambda_{k}(s) d s<\infty \quad P-\text { a.s. }
$$

\footnotetext{
${ }^{14}$ This theorem is adapted from Last and Brandt [1995]
} 
and

$$
G(t)=G(0)+\sum_{k \in \mathbf{K}} \int_{0}^{t} f_{k}(s) d M_{k}(s) \quad P-\text { a.s. }
$$

where the integral is standard Lebesgue-Stieltjes integral.

See Last and Brandt [1995, p.343] for the proof. 